\title{
Stability and boundedness of solutions of the initial value problem for a class of time-fractional diffusion equations
}

\author{
Yanhua Wen ${ }^{*}$, Xian-Feng Zhou and Jun Wang
}

\section{"Correspondence:}

wenyanhua0405@163.com School of Mathematical Sciences, Anhui University, Hefei, 230601 China

\section{Springer}

\begin{abstract}
The aim of this paper is to study the stability and boundedness of solutions of the initial value problem for a class of time-fractional diffusion equations. We first establish a fractional Duhamel principle for the nonhomogeneous time-fractional diffusion equation. Then based on it and the superposition principle, the solution of the above initial value problem is represented. Finally, we obtain the stability and boundedness of the solution and present an illustrative example.
\end{abstract}

Keywords: stability; boundedness; fractional Duhamel principle; initial value problem; time-fractional diffusion equation; Caputo derivative 
ues and delays:

$$
\begin{aligned}
& { }^{C} D_{t}^{\alpha} u(x, t)-a(t) u_{x x}(x, t) \\
& \quad=g\left(t, u\left(x, \tau_{1}(t)\right), u\left(x, \tau_{2}(t)\right), \ldots, u\left(x, \tau_{l}(t)\right)\right), \quad(x, t) \in \Omega \times R_{+}, \\
& u(x, t)=0, \quad(x, t) \in \partial \Omega \times R_{+}, \quad u(x, 0)=\varphi(x), \quad x \in \Omega,
\end{aligned}
$$

where $0 \leq \tau_{i}(t) \leq t, t \in R_{+}(i=1,2, \ldots, l), l$ is a positive integer number, $a(t): R_{+} \rightarrow R$ is continuous and $\varphi(x) \in L^{2}(\Omega) .{ }^{C} D_{t}^{\alpha}$ is the standard Caputo fractional derivative of order $\alpha$ $(0<\alpha \leq 1)$.

In [18], Umarov generalized the classical Duhamel principle for the Cauchy problem to general inhomogeneous fractional distributed differential-operator equations of the form

$$
\begin{aligned}
& L^{\Lambda}[u] \equiv \int_{0}^{\mu} f(\alpha, A) D_{*}^{\alpha} u(t) d \Lambda(\alpha)=h(t), \quad t>0, \\
& u^{(k)}(0)=\varphi_{k}, \quad k=0, \ldots, m-1,
\end{aligned}
$$

where $\mu \in(m-1, m], h(t)$ and $\varphi_{k}, k=0,1, \ldots, m$ are given $X$-valued vector-functions. $D_{*}^{\alpha}$ denotes the operator of fractional differentiation of order $0<\alpha<1$ in the sense of Caputo.

The stability of the solution of a definite solution problem is of great importance in the theory of partial differential equations. However, we have not found any related references which investigate the stability of solutions of initial value problems for time-fractional diffusion equations. Motivated by this fact, in this paper we establish a fractional Duhamel principle, then apply it to study the stability and boundedness of the solution of the timefractional diffusion equation

$$
{ }_{0}^{C} D_{t}^{\alpha} u(x, t)-a^{2} u_{x x}(x, t)=h(x, t), \quad 0<\alpha<1, x \in R, t>0,
$$

with the initial condition

$$
u(x, 0)=\varphi(x), \quad x \in R
$$

where $a \neq 0, \varphi(x) \in L^{p}(R), p \geq 1 . h(x, t)$ is a continuously differentiable function and $h(x, 0)=0 .{ }_{0}^{C} D_{t}^{\alpha}$ represents the following Caputo fractional derivative of order $\alpha>0$ :

$$
{ }_{0}^{C} D_{t}^{\alpha} u(x, t)= \begin{cases}\frac{1}{\Gamma(n-\alpha)} \int_{0}^{t}(t-\tau)^{n-\alpha+1} \frac{\partial^{n} u(x, \tau)}{\partial \tau^{n}} d \tau, & n-1<\alpha<n, \\ \frac{\partial^{n} u(x, t)}{\partial t^{n}}, & \alpha=n,\end{cases}
$$

where $\Gamma$ is the Gamma function and $n=[\alpha]$ denotes the integer part of $\alpha$. Moreover, the Caputo fractional derivative of $\alpha$ is also defined as $\frac{\partial^{\alpha} u(x, t)}{\partial t^{\alpha}}={ }_{0} I_{t}^{n-\alpha} \frac{\partial^{n}}{\partial t^{n}} u(x, t)$.

The rest of this article is organized as follows. Section 2 is devoted to some preliminaries. In Section 3, we present our main results of this paper. An illustrative example is provided in Section 4.

\section{Preliminaries}

In this section, we introduce some definitions and lemmas which will be used later. 
Definition 2.1 ([1]) The two-parameter Mittag-Leffler function is defined as

$$
E_{\alpha, \beta}(z)=\sum_{k=0}^{\infty} \frac{z^{k}}{\Gamma(\alpha k+\beta)}, \quad \alpha, \beta>0,
$$

where $\Gamma$ is the Gamma function.

The Laplace transform of the Mittag-Leffler function in two parameters is

$$
L\left[t^{\alpha k+\beta-1} E_{\alpha, \beta}^{(k)}\left( \pm a t^{\alpha}\right) ; s\right]=\frac{k ! s^{\alpha-\beta}}{\left(s^{\alpha} \mp a\right)^{k+1}}, \quad \operatorname{Re}(s)>|a|^{\frac{1}{\alpha}} .
$$

Definition 2.2 ([1]) The Laplace transform of the Caputo fractional derivative ${ }_{0}^{C} D_{t}^{\alpha} f(t)$ is

$$
\begin{aligned}
L\left[{ }_{0}^{C} D_{t}^{\alpha} f(t) ; s\right] & =\int_{0}^{+\infty} e^{-s t}\left({ }_{0}^{C} D_{t}^{\alpha} f(t)\right) d t \\
& =s^{\alpha} \tilde{f}(s)-\sum_{k=0}^{n-1} s^{\alpha-k-1} f^{(k)}(0), \quad n-1<\alpha \leq n,
\end{aligned}
$$

where $\tilde{f}(s)$ is the Laplace transform of $f(t)$.

Particularly, for $0<\alpha \leq 1$,

$$
L\left[{ }_{0}^{C} D_{t}^{\alpha} f(t) ; s\right]=s^{\alpha} \tilde{f}(s)-s^{\alpha-1} f(0) .
$$

Definition 2.3 ([1]) The Fourier transform of a continuous function $h(x)$ absolutely integrable in $R$ is defined by

$$
\hat{h}(\xi)=F\{h(x) ; \xi\}=\int_{R} e^{i \xi x} h(x) d x, \quad \xi \in R,
$$

and the inverse Fourier transform is defined by

$$
h(x)=F^{-1}\{\hat{h}(\xi) ; x\}=\frac{1}{2 \pi} \int_{R} e^{-i \xi x} \hat{h}(\xi) d \xi, \quad x \in R .
$$

Lemma 2.1 ([18]) Suppose $v(t, \tau)$ is an X-valued function defined for all $t \geq \tau \geq 0$, the derivatives $\frac{\partial^{j} v(t, \tau)}{\partial t^{j}}, 0 \leq j \leq k-1$, are jointly continuous in the $X$-norm, and $\frac{\partial^{k} v(t, \tau)}{\partial t^{k}} \in$ $L^{1}(0, t ; X)$ for all $t>0$. Let $u(t)=\int_{0}^{t} v(t, \tau) d \tau$. Then

$$
\frac{d^{k}}{d t^{k}} u(t)=\sum_{j=0}^{k-1} \frac{d^{j}}{d t^{j}}\left[\left.\frac{\partial^{k-1-j}}{\partial t^{k-1-j}} v(t, \tau)\right|_{\tau=t}\right]+\int_{0}^{t} \frac{\partial^{k}}{\partial t^{k}} v(t, \tau) d \tau .
$$

Lemma 2.2 ([19]) For every $\alpha \in(0,1)$, the uniform estimate

$$
\frac{1}{1+\Gamma(1-\alpha) x} \leq E_{\alpha}(-x) \leq \frac{1}{1+[\Gamma(1+\alpha)]^{-1} x}
$$

holds over $R^{+}$, where $E_{\alpha}(-x)$ denotes $E_{\alpha, 1}(-x)$.

Remark 2.1 Obviously, $0<E_{\alpha, 1}(-x)<1$, for any $x>0$ by Lemma 2.2. 
Lemma 2.3 ([20]) Let $0<\alpha<1$. Then

$$
{ }_{0} I_{t}^{\alpha}\left({ }_{0}^{C} D_{t}^{\alpha} f(t)\right)=f(t)-f(0) .
$$

Lemma 2.4 ([21]) The Fourier transform of the Dirac delta function $\delta(x)$ is

$$
F\{\delta(x) ; \xi\}=\int_{R} e^{i \xi x} \delta(x) d x=1
$$

and the inverse Fourier transform of the Dirac delta function $\delta(x)$ is

$$
\delta(x)=F^{-1}\{1\}=\frac{1}{2 \pi} \int_{R} e^{-i \xi x} d x .
$$

Lemma 2.5 ([21]) The Dirac delta function $\delta(x)$ has the following property:

$$
\int_{R} \delta(x) d x=1
$$

Lemma 2.6 ([21], Hausdorff-Young inequality) If $\in \in L^{1}, g \in L^{p}(p \geq 1)$, then $h=f * g \in L^{p}$ and

$$
\|h\|_{L^{p}} \leq\|f\|_{L^{1}} \cdot\|g\|_{L^{p}}
$$

where $f * g=\int_{R} f(x-y) g(y) d y$ denotes the convolution between $f$ and $g$.

\section{Main results}

In this section, we first consider the situation of $h(x, t)=0$ in the IVP (5)-(6). That is, we discuss the homogeneous IVP

$$
\begin{aligned}
& { }_{0}^{C} D_{t}^{\alpha} u(x, t)-a^{2} u_{x x}(x, t)=0, \quad 0<\alpha<1, x \in R, t>0, \\
& u(x, 0)=\varphi(x), \quad x \in R .
\end{aligned}
$$

Lemma 3.1 The solution of the homogeneous the IVP (21)-(22) has the form

$$
u(x, t)=\int_{R} G(x-y, t) \varphi(y) d y
$$

where $G(x, t)=\frac{1}{2 \pi} \int_{R} e^{-i \xi x} E_{\alpha, 1}\left(-a^{2} \xi^{2} t^{\alpha}\right) d \xi$ is the Green function.

Proof Applying the Laplace transform to equation (21) with respect to the variable $t$ yields

$$
s^{\alpha} \tilde{u}(x, s)-s^{\alpha-1} \varphi(x)-a^{2} \tilde{u}_{x x}(x, s)=0,
$$

then applying the Fourier transform with respect to variable $x$, we obtain

$$
s^{\alpha} \hat{\tilde{u}}(\xi, s)-s^{\alpha-1} \hat{\varphi}(\xi)-a^{2}(-i \xi)^{2} \hat{\tilde{u}}(\xi, s)=0,
$$


where $i^{2}=-1$. So we have

$$
\hat{\tilde{u}}(\xi, s)=\frac{s^{\alpha-1}}{s^{\alpha}+a^{2} \xi^{2}} \hat{\varphi}(\xi)=\frac{s^{\alpha-1}}{s^{\alpha}-\left(-a^{2} \xi^{2}\right)} \hat{\varphi}(\xi) .
$$

Applying the inverse Laplace transform yields

$$
\hat{u}(\xi, t)=E_{\alpha, 1}\left(-a^{2} \xi^{2} t^{\alpha}\right) \hat{\varphi}(\xi)
$$

Furthermore, by using the inverse Fourier transform and Fubini's theorem, we get

$$
\begin{aligned}
u(x, t) & =\frac{1}{2 \pi} \int_{R} e^{-i \xi x} E_{\alpha, 1}\left(-a^{2} \xi^{2} t^{\alpha}\right) \hat{\varphi}(\xi) d \xi \\
& =\frac{1}{2 \pi} \int_{R} e^{-i \xi x} E_{\alpha, 1}\left(-a^{2} \xi^{2} t^{\alpha}\right) \int_{R} e^{i \xi y} \varphi(y) d y d \xi \\
& =\int_{R} \frac{1}{2 \pi} \int_{R} e^{-i \xi(x-y)} E_{\alpha, 1}\left(-a^{2} \xi^{2} t^{\alpha}\right) d \xi \varphi(y) d y \\
& =\int_{R} G(x-y, t) \varphi(y) d y,
\end{aligned}
$$

where

$$
G(x, t)=\frac{1}{2 \pi} \int_{R} e^{-i \xi x} E_{\alpha, 1}\left(-a^{2} \xi^{2} t^{\alpha}\right) d \xi
$$

is the Green function. This completes the proof.

Property 3.1 The Green function $G(x, t)$ has the following property:

$$
\int_{R} G(x, t) d x<1, \quad t>0 .
$$

Proof By Lemma 2.2, it follows

$$
\begin{aligned}
G(x, t) & =\frac{1}{2 \pi} \int_{R} e^{-i \xi x} E_{\alpha, 1}\left(-a^{2} \xi^{2} t^{\alpha}\right) d \xi \\
& \leq \frac{1}{2 \pi}\left|\int_{R} e^{-i \xi x} E_{\alpha, 1}\left(-a^{2} \xi^{2} t^{\alpha}\right) d \xi\right| \\
& \leq \frac{1}{2 \pi} \int_{R} e^{-i \xi x}\left|E_{\alpha, 1}\left(-a^{2} \xi^{2} t^{\alpha}\right)\right| d \xi \\
& <\frac{1}{2 \pi} \int_{R} e^{-i \xi x} \cdot 1 d \xi \\
& =\delta(x) .
\end{aligned}
$$

Lemma 2.5 implies that

$$
\int_{R} G(x, t) d x<\int_{R} \delta(x) d x=1, \quad t>0
$$

which completes the proof. 


\subsection{Fractional Duhamel principle}

We now consider equation (5) with the initial data $u(x, 0)=\varphi(x)=0$. That is, we study the nonhomogeneous IVP

$$
\begin{aligned}
& { }_{0}^{C} D_{t}^{\alpha} u(x, t)-a^{2} u_{x x}(x, t)=h(x, t), \quad 0<\alpha<1, x \in R, t>0, \\
& u(x, 0)=0, \quad x \in R .
\end{aligned}
$$

A fractional Duhamel principle is firstly given, which can reduce the nonhomogeneous the IVP (33)-(34) to the corresponding homogeneous IVP.

Theorem 3.1 (Fractional Duhamel principle) The solution of the nonhomogeneous the IVP (33)-(34) is given by

$$
u(x, t)=\int_{0}^{t} w(x, t ; \tau) d \tau
$$

where $w(x, t ; \tau)$ is the solution of the homogeneous equation

$$
{ }_{0}^{C} D_{t}^{\alpha} w(x, t)-a^{2} w_{x x}(x, t)=0, \quad 0<\alpha<1, x \in R, t>\tau,
$$

satisfying

$$
t=\tau: w(x, \tau)={ }_{0}^{C} D_{\tau}^{1-\alpha} h(x, \tau)
$$

where $h(x, t)$ is a continuously differentiable function.

Proof Assume that $w(x, t ; \tau)$ is the solution of the IVP (36)-(37). We next prove that $u(x, t)=\int_{0}^{t} w(x, t ; \tau) d \tau$ is the solution of the IVP (33)-(34). Let $k=1$ in Lemma 2.1, then

$$
\frac{\partial}{\partial t} u(x, t)=\left.w(x, t ; \tau)\right|_{\tau=t}+\int_{0}^{t} \frac{\partial}{\partial t} w(x, t ; \tau) d \tau
$$

Thus it follows that

$$
\begin{aligned}
{ }_{0}^{C} & D_{t}^{\alpha} u(x, t)-a^{2} u_{x x}(x, t) \\
& ={ }_{0} I_{t}^{1-\alpha} \frac{\partial}{\partial t} u(x, t)-a^{2} u_{x x}(x, t) \\
& ={ }_{0} I_{t}^{1-\alpha} \frac{\partial}{\partial t} \int_{0}^{t} w(x, t ; \tau) d \tau-\int_{0}^{t} a^{2} w_{x x}(x, t ; \tau) d \tau \\
& ={ }_{0} I_{t}^{1-\alpha}\left[\left.w(x, t ; \tau)\right|_{\tau=t}+\int_{0}^{t} \frac{\partial}{\partial t} w(x, t ; \tau) d \tau\right]-\int_{0}^{t} a^{2} w_{x x}(x, t ; \tau) d \tau \\
& ={ }_{0} I_{t}^{1-\alpha}\left({ }_{0}^{C} D_{t}^{1-\alpha} h(x, t)\right)+\int_{0}^{t}\left[{ }_{0} I_{t}^{1-\alpha} \frac{\partial}{\partial t} w(x, t ; \tau)-a^{2} w_{x x}(x, t ; \tau)\right] d \tau \\
& =h(x, t)-h(x, 0)+\int_{0}^{t}\left[{ }_{0}^{C} D_{t}^{\alpha} w(x, t ; \tau)-a^{2} w_{x x}(x, t ; \tau)\right] d \tau \\
& =h(x, t) .
\end{aligned}
$$


In addition, $u(x, 0)=0$. Therefore, $u(x, t)=\int_{0}^{t} w(x, t ; \tau) d \tau$ is the solution of the IVP (33)(34). The proof is completed.

Corollary 3.1 (i) The the IVP (36)-(37) has the solution. In fact, let $t^{\prime}=t-\tau$ in (36)-(37), then the IVP (36)-(37) can be turned into the form

$$
\begin{aligned}
& { }_{0}^{C} D_{t}^{\alpha} w\left(x, t^{\prime} ; \tau\right)-a^{2} w_{x x}\left(x, t^{\prime} ; \tau\right)=0, \quad 0<\alpha<1, x \in R, t^{\prime}>0, \\
& t^{\prime}=0: w(x, 0 ; \tau)={ }_{0}^{C} D_{\tau}^{1-\alpha} h(x, \tau), \quad x \in R .
\end{aligned}
$$

Lemma 3.1 implies that the solution of the problem (40)-(41) can be obtained by

$$
w\left(x, t^{\prime} ; \tau\right)=\int_{R} G\left(x-y, t^{\prime}\right)_{0}^{C} D_{\tau}^{1-\alpha} h(y, \tau) d y .
$$

Hence, the solution of the IVP (36)-(37) can be represented as

$$
w(x, t-\tau ; \tau)=\int_{R} G(x-y, t-\tau)_{0}^{C} D_{\tau}^{1-\alpha} h(y, \tau) d y .
$$

(ii) Furthermore, by Theorem 3.1, the solution of the IVP (33)-(34) has the form

$$
u(x, t)=\int_{0}^{t} w(x, t-\tau ; \tau) d \tau=\int_{0}^{t} \int_{R} G(x-y, t-\tau)_{0}^{C} D_{\tau}^{1-\alpha} h(y, \tau) d y d \tau
$$

Combining Lemma 3.1 with Corollary 3.1, we can get the following theorem.

Theorem 3.2 The solution of the nonhomogeneous the IVP (5)-(6) has the form

$$
u(x, t)=u_{1}(x, t)+u_{2}(x, t)
$$

where $u_{1}(x, t), u_{2}(x, t)$ are solutions of the IVPs (21)-(22), (33)-(34), respectively. That is,

$$
u(x, t)=\int_{R} G(x-y, t) \varphi(y) d y+\int_{0}^{t} \int_{R} G(x-y, t-\tau)_{0}^{C} D_{\tau}^{1-\alpha} h(y, \tau) d y d \tau
$$

where $G(x, t)=\frac{1}{2 \pi} \int_{R} e^{-i \xi x} E_{\alpha, 1}\left(-a^{2} \xi^{2} t^{\alpha}\right) d \xi$ is the Green function.

Theorem 3.3 When $t \rightarrow 0$, the solution (46) of the Cauchy problem (5)-(6) is bounded by the initial data

$$
\|u(x, t)\|_{L^{p}(R)} \leq\|u(x, 0)\|_{L^{p}(R)}, \quad x \in R, p \geq 1
$$

Proof From (46) and Lemma 2.4, we have

$$
\begin{aligned}
\lim _{t \rightarrow 0}\|u(x, t)\|_{L^{p}(R)} & =\left\|\int_{R} G(x-y, 0) u(y, 0) d y\right\|_{L^{p}(R)} \\
& =\left\|\int_{R} \delta(x-y) u(y, 0) d y\right\|_{L^{p}(R)} \\
& =\|\delta(x) * u(x, 0)\|_{L^{p}(R)}, \quad p \geq 1 .
\end{aligned}
$$


Then the inequality (48), Lemma 2.6 and the property of the Dirac delta function $\delta(x)$ imply

$$
\begin{aligned}
\lim _{t \rightarrow 0}\|u(x, t)\|_{L^{p}(R)} & =\|\delta(x) * u(x, 0)\|_{L^{p}(R)} \\
& \leq\|\delta(x)\|_{L^{1}(R)} \cdot\|u(x, 0)\|_{L^{p}(R)} \\
& \leq\|u(x, 0)\|_{L^{p}(R)}
\end{aligned}
$$

for $p \geq 1$, which completes the proof.

\subsection{Stability of solution}

This section presents the stability of the solution of the nonhomogeneous the IVP (5)-(6).

Definition 3.1 Suppose that $H$ is a linear normed space with the norm $\|\cdot\|_{H}, u_{1}(x, t)$, $u_{2}(x, t)$ are solutions of the IVP (5)-(6) corresponding to initial datum $\varphi_{1}(x), \varphi_{2}(x)$, respectively. For any $\varepsilon>0$, if there exists a constant $\delta>0$ such that $\left\|\varphi_{1}(x)-\varphi_{2}(x)\right\|<$ $\delta$ implies $\left\|u_{1}(x, t)-u_{2}(x, t)\right\|<\varepsilon$, then we say that the solution of the IVP (5)-(6) is stable.

Theorem 3.4 (Stability) Assume $\varphi(x) \in L^{p}(R), p \geq 1$. Then the solution $u(x, t)$ of the nonhomogeneous IVP (5)-(6) is stable.

Proof Suppose that $u_{1}(x, t)$ is the solution of the nonhomogeneous IVP

$$
\begin{aligned}
& { }_{0}^{C} D_{t}^{\alpha} u(x, t)-a^{2} u_{x x}(x, t)=h(x, t), \quad 0<\alpha<1, x \in R, t>0, \\
& u(x, 0)=\varphi_{1}(x), \quad x \in R
\end{aligned}
$$

and that $u_{2}(x, t)$ is the solution of the nonhomogeneous IVP

$$
\begin{aligned}
& { }_{0}^{C} D_{t}^{\alpha} u(x, t)-a^{2} u_{x x}(x, t)=h(x, t), \quad 0<\alpha<1, x \in R, t>0, \\
& u(x, 0)=\varphi_{2}(x), \quad x \in R .
\end{aligned}
$$

Then the superposition principle implies that $u_{1}(x, t)-u_{2}(x, t)$ is the solution of the following homogeneous IVP:

$$
\begin{aligned}
& { }_{0}^{C} D_{t}^{\alpha} u(x, t)-a^{2} u_{x x}(x, t)=0, \quad 0<\alpha<1, x \in R, t>0, \\
& u(x, 0)=\varphi_{1}(x)-\varphi_{2}(x), \quad x \in R .
\end{aligned}
$$

By Lemma 3.1, we get

$$
u_{1}(x, t)-u_{2}(x, t)=\int_{R} G(x-y, t)\left[\varphi_{1}(y)-\varphi_{2}(y)\right] d y,
$$


where $G(x, t)=\frac{1}{2 \pi} \int_{R} e^{-i \xi x} E_{\alpha, 1}\left(-a^{2} \xi^{2} t^{\alpha}\right) d \xi$ is the Green function. Taking the $L^{p}$-norm $(p \geq$ 1) on both sides of equation (56), then Lemma 2.6 yields

$$
\begin{aligned}
\left\|u_{1}(x, t)-u_{2}(x, t)\right\|_{L^{p}(R)} & =\left\|\int_{R} G(x-y, t)\left[\varphi_{1}(y)-\varphi_{2}(y)\right] d y\right\|_{L^{p}(R)} \\
& =\left\|G(x, t) *\left[\varphi_{1}(x)-\varphi_{2}(x)\right]\right\|_{L^{p}(R)} \\
& \leq\|G(x, t)\|_{L^{1}(R)} \cdot\left\|\varphi_{1}(x)-\varphi_{2}(x)\right\|_{L^{p}(R)}
\end{aligned}
$$

for $t>0$. Then from Lemma 2.6 and the property of the Green function $G(x, t)$ it follows that

$$
\begin{aligned}
\left\|u_{1}(x, t)-u_{2}(x, t)\right\|_{L^{p}(R)} & \leq\|G(x, t)\|_{L^{1}(R)} \cdot\left\|\varphi_{1}(x)-\varphi_{2}(x)\right\|_{L^{p}(R)} \\
& <\left\|\varphi_{1}(x)-\varphi_{2}(x)\right\|_{L^{p}(R)}, \quad t>0 .
\end{aligned}
$$

For any $\varepsilon>0$, choose $\delta<\varepsilon$. Then $\left\|\varphi_{1}(x)-\varphi_{2}(x)\right\|_{L^{p}(R)}<\delta$ implies $\left\|u_{1}(x, t)-u_{2}(x, t)\right\|_{L^{p}(R)}<$ $\varepsilon, t>0$. By Definition 3.1, the solution $u(x, t)$ of the nonhomogeneous the IVP (5)-(6) is stable. The proof is completed.

\section{Illustrative example}

In this section, we provide an example to show the application of our stability result.

Example 4.1 Consider the following nonhomogeneous equation:

$$
{ }_{0}^{C} D_{t}^{\alpha} u(x, t)-a^{2} \frac{\partial^{2} u(x, t)}{\partial x^{2}}=2 t^{1-\alpha}\left(x^{2}+x+1\right), \quad 0<\alpha<1, x \in R, t>0,
$$

with the initial condition

$$
u(x, 0)=x^{2}, \quad x \in R
$$

The well-known formula $(0<\alpha<1)$

$$
{ }^{C} D_{t}^{\alpha} t^{\beta}=\frac{\Gamma(\beta+1)}{\Gamma(\beta-\alpha+1)} t^{\beta-\alpha}, \quad \beta>0,
$$

and Theorem 3.2 imply that the solution of the IVP (59)-(60) is

$$
u(x, t)=\int_{R} G(x-y, t) y^{2} d y+2 \Gamma(2-\alpha) \int_{0}^{t} \int_{R} G(x-y, t-\tau)\left(y^{2}+y+1\right) d y d \tau .
$$

Suppose that $u_{1}(x, t), u_{2}(x, t)$ are solutions of the IVP (59)-(60) corresponding to initial datum $x_{1}^{2}, x_{2}^{2}$, respectively. Then, for $p \geq 1$, we have

$$
\begin{aligned}
\left\|u_{1}(x, t)-u_{2}(x, t)\right\|_{L^{p}(R)} & \leq\|G(x, t)\|_{L^{1}(R)} \cdot\left\|x_{1}^{2}-x_{2}^{2}\right\|_{L^{p}(R)} \\
& <\left\|x_{1}^{2}-x_{2}^{2}\right\|_{L^{p}(R)}, \quad t>0 .
\end{aligned}
$$

For any $\varepsilon>0$, choose $\delta<\varepsilon$. Then $\left\|x_{1}^{2}-x_{2}^{2}\right\|_{L^{p}(R)}<\delta$ implies $\left\|u_{1}(x, t)-u_{2}(x, t)\right\|_{L^{p}(R)}<\varepsilon, t>0$. According to Definition 3.1, the solution (62) of the IVP (59)-(60) is stable. 


\section{Acknowledgements}

This paper is supported by National Natural Science Foundation of China (11371027, 11471015 and 11601003), Natural Science Foundation of Anhui Province (1508085MA01, 1608085MA12 and 1708085MA15) and Program of Natural Science Research for Universities of Anhui Province (KJ2016A023).

\section{Competing interests}

The authors declare that they have no competing interests.

\section{Authors' contributions}

All authors contributed equally in this article. They read and approved the final manuscript.

\section{Publisher's Note}

Springer Nature remains neutral with regard to jurisdictional claims in published maps and institutional affiliations.

Received: 9 January 2017 Accepted: 10 July 2017 Published online: 09 August 2017

\section{References}

1. Podlubny, I: Fractional Differential Equations. Academic Press, New York (1993)

2. Agarwal, RP, Zhou, Y, He, YY: Existence of fractional neutral functional differential equations. Comput. Math. Appl. 59, 1095-1100 (2010)

3. Zhou, XF, Hu, LG, Liu, S, Jiang, W: Stability criterion for a class of nonlinear fractional differential systems. Appl. Math. Lett. 28, 25-29 (2014)

4. Zhou, XF, Huang, Q, Jiang, W: A note on the stability criterion for a class of nonlinear fractional differential systems. Appl. Math. Lett. 31, 16-17 (2014)

5. Liu, KW, Jiang, W: Stability of nonlinear Caputo fractional differential equations. Appl. Math. Model. 40, 3919-3924 (2016)

6. Jleli, M, Kirane, M, Samet, B: Lyapunov-type inequality for fractional partial differential equations. Appl. Math. Lett. 66, 30-39 (2017)

7. Luchko, Y: Maximum principle for the generalized time-fractional diffusion equation. J. Math. Anal. Appl. 351, 218-223 (2009)

8. Rawashdeh, MS, Jammal, HA: New approximate solutions to fractional nonlinear systems of partial differential equations using the FNDM. Adv. Differ. Equ. 2016, 235 (2016)

9. Ferreira, M, Vieira, N: Fundamental solutions of the time fractional diffusion-wave and parabolic Dirac operators. J. Math. Anal. Appl. 447, 329-353 (2017)

10. Morales-Delgado, VF, Gómez-Aguilar, JF: Laplace homotopy analysis method for solving linear partial differential equations using a fractional derivative with and without kernel singular. Adv. Differ. Equ. 2016, 164 (2016)

11. Liu, YJ: Existence and uniqueness of solutions for a class of initial value problems of fractional differential systems on half lines. Bull. Sci. Math. 137, 1048-1071 (2013)

12. Kosmatov, N: Integral equations and initial value problems for nonlinear differential equations of fractional order. Nonlinear Anal. 70, 2521-2529 (2009)

13. Agarwal, RP, Benchohra, M, Hamani, A: Boundary value problem for fractional differential equations. Georgian Math. J. $16,401-411(2009)$

14. Seemab, A, Rehman, M: A note on fractional Duhamel's principle and its application to a class of fractional partial differential equations. Appl. Math. Lett. 64, 8-14 (2017)

15. Wang, JR, Zhang, Y: Nonlocal initial value problems for differential equations with Hilfer fractional derivative. Appl. Math. Comput. 266, 850-859 (2015)

16. Zhu, B, Liu, L, Wu, Y: Local and global existence of mild solutions for a class of nonlinear fractional reaction-diffusion equations with delay. Appl. Math. Lett. 61, 73-79 (2016)

17. Ouyang, Z: Existence and uniqueness of the solutions for a class of nonlinear fractional order partial differentia equations with delay. Comput. Math. Appl. 61, 860-870 (2011)

18. Umarov, S: On fractional Duhamel's principle and its applications. J. Differ. Equ. 252, 5217-5234 (2012)

19. Simon, T: Comparing Fréchet and positive stable laws (2014). arXiv:1310.1888v2 [math.PR]

20. Kilbas, AA, Srivastava, HM, Trujillo, JJ: Theory and Applications of Fractional Differential Equations. Elsevier, Amsterdam (2006)

21. Hörmander, L: The Analysis of Linear Partial Differential Operators. Springer, Berlin (2005) 